\title{
Applications of Self-Assembled Monolayers in Surface-Enhanced Raman Scattering
}

\author{
Charles K. Klutse, Adam Mayer, Julia Wittkamper, and Brian M. Cullum \\ Department of Chemistry and Biochemistry, University of Maryland, Baltimore County, 1000 Hilltop Circle, \\ Baltimore, MD 21250, USA \\ Correspondence should be addressed to Brian M. Cullum, cullum@umbc.edu
}

Received 8 February 2012; Accepted 16 March 2012

Academic Editor: Mustafa Çulha

Copyright (๑) 2012 Charles K. Klutse et al. This is an open access article distributed under the Creative Commons Attribution License, which permits unrestricted use, distribution, and reproduction in any medium, provided the original work is properly cited.

The increasing applications of surface-enhanced Raman scattering (SERS) has led to the development of various SERS-active platforms (SERS substrates) for SERS measurement. This work reviews the current optimization techniques available for improving the performance of some of these SERS substrates. The work particularly identifies self-assembled-monolayer- (SAM-) based substrate modification for optimum SERS activity and wider applications. An overview of SERS, SAM, and studies involving SAM-modified substrates is highlighted. The focus of the paper then shifts to the use of SAMs to improve analytical applications of SERS substrates by addressing issues including long-term stability, selectivity, reproducibility, and functionalization, and so forth. The paper elaborates on the use of SAMs to achieve optimum SERS enhancement. Specific examples are based on novel multilayered SERS substrates developed in the author's laboratory where SAMs have been demonstrated as excellent dielectric spacers for improving SERS enhancement more than 20-fold relative to conventional single layer SERS substrates. Such substrate optimization can significantly improve the sensitivity of the SERS method for analyte detection.

\section{Introduction}

There is a great interest in the development of SERS-based analytical techniques for real-time monitoring of intracellular events due to the advantages SERS has compared to other commonly used optical techniques in biological analyses. SERS provides narrow spectral bands, thereby allowing multiplex detection of analytes in complex sampling environments. It also has potential for quenching autofluorescence that can interfere with SERS measurements. SERS is the enhancement of Raman intensity of analytes which are in close proximity to nanoscaled roughened metallic surfaces (normally referred to as SERS substrates) $[1,2]$. The ratio of such Raman scattering intensities of a given number of analyte molecules to the intensities of the same number of molecules in the absence of the SERS substrates is the SERS enhancement factor (EF) [3]. SERS EF is a widely accepted parameter for estimating the SERS activity of the SERS substrates, with larger SERS EFs indicating highly SERS-active substrates. While the commonly reported SERS
EFs can range from $10^{6}$ to $10^{8}$, SERS EF can reach $10^{14}$ on certain special substrates, making SERS capable of single molecule detection [4-6].

In recent years, SERS has been demonstrated as a powerful tool for a wide range of analyses. Critical to the quality of the SERS analyses is the characteristic of the SERS substrates on which the SERS is conducted. As a result of this, the development and optimization of the SERS substrate for routine analytical purposes has become a very attractive area of research. In this paper, the recent development on SERS substrates involving the use of SAMs for various forms of modifications is discussed. The discussion begins with an overview of SERS and its analytical importance, followed by the identification of a broad category of SERS substrates that are commonly employed for SERS measurements. A brief description of SAM and how it features in SERS is given. The main subject for the paper then focuses on the use of thiolated organic molecules for the optimization of SERS. The discussion on SAM-based optimization of SERS will involve the modification of the 
SERS-active surface with SAMs to improve selectivity, longterm stability, reproducibility, substrate functionalization, and so forth. The paper will also discuss the SAM-based multilayer SERS substrate optimization for the enhancement of SERS activity. The area of SERS substrate development is extensive and cannot be exhausted in this paper. Therefore, the paper will focus on some of the most commonly used SERS substrates which include metal colloids and metalcoated nanostructures.

\section{Overview of SERS}

Raman spectroscopy has various analytical applications, especially in research fields requiring high analyte specificity, because it is capable of providing molecular structural information about analytes of interest without the need for exogenous labels. Additionally, as a result of the weak Raman scattering of water, Raman spectroscopy can be applied to aqueous complex biological samples with little or no sample preparation, making it an ideal analytical tool for monitoring biomolecules within individual living cells. However, Raman lacks the sensitivity for the detection of analytes at ultratrace level due to extremely small Raman cross-section. In 1974, Fleischmann and coworkers observed very intense Raman scattering of pyridine adsorbed on electrochemically roughened silver electrodes, which was attributed to the large surface area of the roughened electrode $[7,8]$. Later, Jeanmaire and Van Duyne and Albrecht and Creighton working independently observed that the enhanced Raman scattering on the roughened surface exceeds what is expected from the increase in surface area arising from the roughening of the electrode. Their work led to the proposal of SERS mechanisms (broadly categorized as electromagnetic (EM) and chemical mechanisms) to explain the enhancement of Raman scattering on roughened metal surfaces $[9,10]$. Thus, the phenomenon of achieving enhanced Raman scattering on specialized surfaces became known as SERS. Importantly, it became clear that other metals including gold and copper can be used as SERS-active surfaces $[11,12]$. By using SERS, it is now possible to achieve high sensitivity in addition to the various Raman advantages, making SERS a powerful tool for a broad range of analyses. In recent years, SERS has been applied in biomedical research, defense and security, diseases diagnosis and prevention, single-cell analysis, and for quantitative detection of analytes in various samples [1326].

\section{SERS Substrates}

The quest for standardized SERS substrates capable of routine SERS-based chemical analysis and the need for deeper understanding of SERS have contributed largely to the advances in SERS substrate development. As a result, since the discovery of SERS, various techniques have been applied for the fabrication of these substrates [27-29]. Progress in SERS substrates development, which is not the focus of this work, has been reviewed elsewhere [27, 30,31], and therefore only a brief overview will be discussed. Electrochemically roughened metal electrodes were the earliest types of SERS substrates. However, advances in nanofabrication have significantly influenced SERS substrates development. Colloidal metal nanoparticles are some of the commonly used SERS substrates. They are normally used as suspensions and their plasmonic properties can easily be modified using their sizes and shapes in order to improve their SERS activities [2, 23, 32-36]. Although colloidal metal nanoparticles are notable for high SERS activities at their junctions, they exhibit large variations in SERS signals due to the random occurrence of these junctions. To minimize such variations, the colloids can sometimes be self-assembled on planar platforms to create relatively regular junctions among the colloids [3639]. Nanolithographic methods are also commonly used to fabricate highly ordered SERS substrates by forming nanostructures directly on a small area of a solid support. Such nanostructures can be achieved by selective etching of the specialized surface using an ultraviolet light or electron beam and then coating the etched surface with a SERS-active metal film [40-42]. Orderly structured SERS substrates can also be derived through a method commonly referred to as nanosphere lithography. In this method, metal films are deposited (using either physical or chemical means) on nanospheres arranged on a solid support. The removal of the nanospheres leaves a regular array of nanostructures, forming the SERS substrates [43-45]. While lithographically produced SERS substrates are highly reproducible, they are difficult and time consuming to produce in mass quantities.

A highly ordered SERS substrate which can easily be produced in mass quantities is based on nanostructures arranged on solid planar support followed by the deposition of a thin layer of metal film on the nanostructures. Unlike nanosphere lithography, it is the metal-coated nanostructures that are used as the SERS substrates, normally referred to as metal film on nanostructures (MFONs). A schematic representation of MFON substrates fabrication is shown in Figure 1. Other unique features of these types of SERS substrates include a combination of the advantages of 3D nanosized metal colloids for probing microsampling environments and the highly ordered and regularly arranged nanostructures on solid platforms for reproducible SERS measurements. Thus, as shown in Figure 1, the metal-coated nanostructured platform can be used as a planar SERS substrate for routine SERS analyses [30, 46, 47]. Importantly, the individual metal film-coated nanospheres can also be used as sensor supports for SERS-based bio-nanosensors, which will be discussed later $[19,26]$.

\section{Self-Assembled Monolayers}

Although SERS substrates with very large SERS EFs have demonstrated the high sensitivity of SERS-based analysis, the bare substrates lack the selectivity that will allow their use in complex samples. However, by using SAMs to modify substrates, it is possible to make the SERS substrates selective as well as tailor other desirable qualities that allow them to be used for various analytical purposes. SAMs are derived from the adsorption of organic molecules onto surfaces of metals, metal oxides, semiconductors, and other platforms, to form a thin layer. SAMs can affect the interface properties 


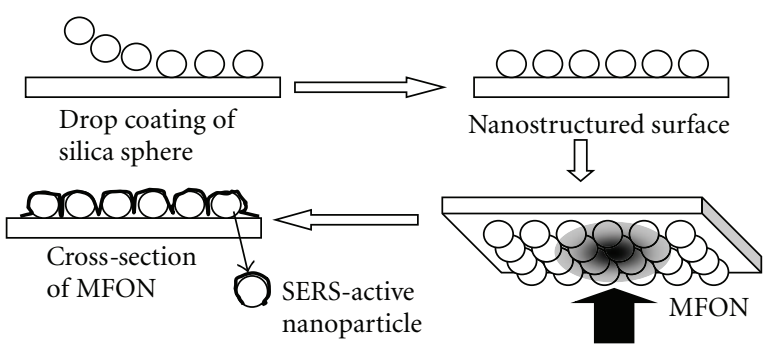

Figure 1: A flow diagram of MFON fabrication.

in many ways including insulation of conducting surfaces, creating hydrophobic surfaces, allowing them to be used for the modification and study of interfacial phenomena [4851]. There are widespread examples of SAMs with equally widespread uses. However, this paper will focus on SAMs involving thiol-modified organic molecules (e.g., alkylthiols) that have been specifically employed in SERS to improve the SERS substrates characteristics. An idealized representation of molecules used for SAM formation is shown schematically in Figure 2. A typical example of a molecule used for SAM formation is made up of a thiol group, which attaches to the substrates, and the hydrocarbon tail which can be varied in order to vary the thickness of the SAM. An essential part of the molecule is the terminal functional group which can be varied in order to confer a specific surface chemistry to suit the intended application of the monolayer.

\section{SAMs Applications in SERS}

SAMs applications in SERS are diverse and include surface chemistry and substrates modifications. The unique attribute of SAMs to affect surfaces and the SERS ability to probe surface chemistry has been exploited as investigative tools in various ways. For example, SAM has been used to verify the EM mechanisms of SERS [52, 53]. Halas et al. used various lengths of labeled DNA self-assembled on SERS substrate as molecular rulers to investigate the EM field effect on SERS. By varying the lengths of SAMs and measuring Raman intensities of the Raman labels positioned at the tail ends of these rulers, it was observed that the shorter chains showed more intense Raman scattering. This was because the labels attached to the shorter chains were nearer to the metal surface and therefore exhibited better interaction with the EM field relative to the longer chain lengths [50, 53-55]. Conversely, SERS has been applied to study SAMs including verifying the mechanism of thiol-metal surface interaction [54, 56-59]. By doing Raman and SERS measurements of several alkylthiols, it was found that the $\mathrm{S}-\mathrm{H}$ vibration at $2575 \mathrm{~cm}^{-1}$ was absent when the molecules adsorbed on the metal surface. However, the Raman band at $200 \mathrm{~cm}^{-1}$ associated with $\mathrm{Ag}-\mathrm{S}$ vibrations appeared, confirming the formation of SAM via the cleavage of the $\mathrm{S}-\mathrm{H}$ bond. Another confirmation of the formation of SAM on SERS substrates is the high intensity of the $\mathrm{C}-\mathrm{S}$ band at $650 \mathrm{~cm}^{-1}$ due to its proximity to the SERS-active surface $[54,60]$.

The most widespread application of SAMs in SERS is their use for SERS substrates modification as shown in

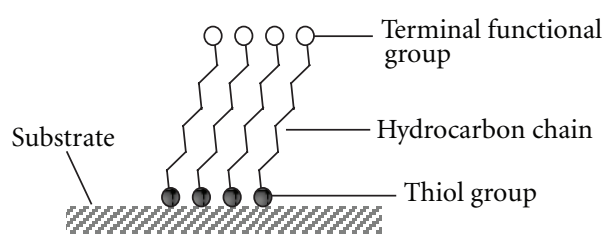

FIGURE 2: Schematic representation of the individual molecules adsorbed on metal substrate to form SAM.

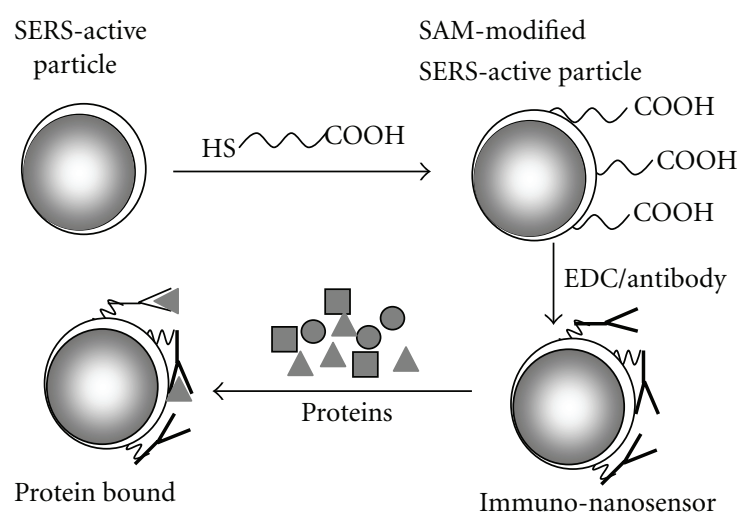

FIGURE 3: Schematic representation of the SERS-based immunonanosensor via - $\mathrm{COOH}$ - terminated SAM for label-free protein detection.

Figure 3. Metal colloids are often protected with SAMs in order to preserve the integrity of the particles and prevent them from aggregating. SAMs have been shown to influence the absorption characteristics of metal colloids. Thus, SAMs can be used to controllably tailor the wavelength of surface plasmon absorption of substrates to match the excitation wavelength, thereby improving the SERS activity of the metal colloids [50, 61]. Functionalization of SERS substrates has been seen as a means of improving analyte specificity and reducing random adsorption of untargeted molecules. SAMs have been employed as cross-linkers primarily to aid functionalization of SERS substrates [55, 62-65]. Used in this way, SAMs with specially functionalized tail groups can be immobilized on the bare SERS substrate before attaching recognition elements for specific analytes $[19,66]$. Culha et al. have used SAM-modified SERS platforms as models for gene diagnostics. In these models, the tail functional group of 1-mercaptohexane was modified with Rhodamine B-labeled single-stranded DNA complementary to breast cancer gene (BRCA1). To prevent the long HS- $\left(\mathrm{CH}_{2}\right)_{6}-$ ssDNA-RhB strands from folding, they were interspaced with 6-mercaptohexanol. The SAM-modified SERS platform was capable of specifically detecting the breast cancer gene by complementary paring [62]. SERS substrates have also been functionalized via SAMs for label-free detection of proteins in modeled environments at physiological concentrations. As shown in Figure 3, the SERS-active nanoparticles which were derived from MFON SERS substrates were first immobilized with carboxylic acid-terminated SAMs. Molecules of antihuman insulin $(\operatorname{IgG})$ were then bound to the SAM via the commonly used EDC chemistry to develop SERS-based 
immuno-nanosensors. The immuno-nanosensors were then used to detect insulin in cell lysates at a concentration of $10 \mu \mathrm{g} / \mathrm{mL}[25,67,68]$.

Applications SAMs in the optimization of SERS substrate have been done specifically to improve various substrates characteristics, including long-term stability, analyte selectivity, and precision across the sampling volume. Due to their unique abilities of influencing interfacial properties of substrates, SAMs can be used to modify the affinity of SERS substrates towards specific analytes, thereby improving their selectivity. For instance, SAM-modified SERS substrates have been used to partition analyte from known matrices and concentrate them on the SERS substrate for detection [6971]. Such substrates have been used as SERS platforms for the detection of polycyclic aromatic hydrocarbons (PAHs). PAHs adsorbed on the bare gold SERS-active surface are known to undergo surface-induced decomposition due to the catalytic properties of the roughened gold surface. By modifying the surface with 1-propanethiol, it was possible to selectively concentrate PAHs on the substrate, reduce decomposition, and improve reproducibility and stability [72]. Apart from improving selectivity, SAMs have been used to increase the long-term stability of the substrates by means of partial coverage of the SERS-active surface with the SAM [73].

One major drawback of SERS is its lack of reproducibility due to the random distribution of the SERS hot spots across the substrate surface. SAMs have been used as forms of internal standards to account for the variations in the SERS signals [74-81]. In one such study, SERS intensities of analytes were taken on SERS substrates modified with labeled SAMs. It was assumed that both the labeled SAM and the analyte experienced the same excitation and substrate conditions during a particular SERS measurement. For this reason, although the SERS intensities of the analytes varied from spot to spot on the SERS substrates, ratios of the labeled SAM intensity to the analyte intensity did not vary significantly. Thus, the SAM is used in this case as an internal standard to normalize the SERS intensity of the analytes. Despite these optimization processes, there are issues concerning the reduction of the SERS effect on the analyte due to the coverage of the SERS active platforms with SAM, effectively reducing the sensitivity of SAM-modified SERS substrates [52].

\section{SAM Multilayer SERS Substrate Optimization}

With the increasing potential application of SERS, the search for optimized SERS substrates that lack the limitations of most conventional SERS substrates has become a priority. In 2005, Cullum's group discovered that MFON SERS substrates can be optimized to achieve larger SERS EFs by using SERS substrates based on multiple layers of SERS-active surfaces separated by dielectric spacers (i.e., metal oxide) [82]. As shown in Figure 4, the first reported multilayer SERS substrate consisted of multiple layers of silver film separated by silver oxide. Using the multilayer geometry, SERS substrates can be optimized without making the SERS-active surface less accessible to analytes. Although it has generally been observed that SERS enhancement is

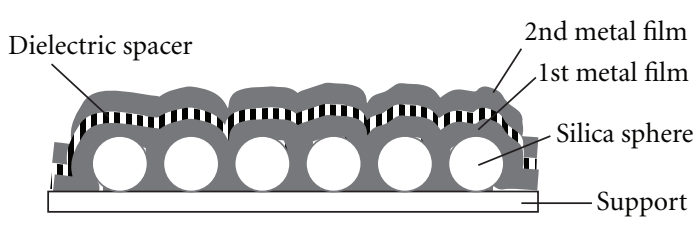

FIGURE 4: An idealized multilayer SERS substrate.

significantly affected by the dielectric constant around the SERS substrate, how this observation relates to the multilayer SERS enhancement is still under investigation. However, it is thought that the optimum interaction of the EM fields generated on the multiple SERS-active surfaces largely contributed to the observed multilayer SERS enhancement. Additionally, the results of the multilayer SERS studies suggested that efficient separation of the SERS-active surfaces, the type of dielectric spacer used, and the thickness of this spacer can significantly affect the multilayer SERS enhancement [83, 84]. For this reason, our laboratory has been investigating other forms of dielectric spacers (e.g., polymers, silica, and SAMs) and SERS-active metal films for the systematic multilayer SERS optimization $[84,85]$.

Systematic optimization of multilayer SERS requires control of the dielectric spacer and a thorough understanding of its effect on the SERS enhancement. Since 2007, data gathered on the optimization of multilayer SERS using SAMs as the main dielectric spacers have generated interesting yet nonsystematic results. To provide a more systematic evaluation, SAMs have recently been employed to vary spacer thickness and dielectric constant in a more controlled fashion, based on the choice of the hydrocarbon chain length and tail functional group, respectively. To form SAM multilayer SERS substrates, a single-layer MFON, referred to as single film on nanostructure (SFON), was immersed in $1 \mathrm{mM}$ solutions containing the appropriate alkylthiol for SAM formation. The substrate was retrieved and rinsed to remove unattached molecules of the spacers. After the removal of the unattached molecules, a second layer of metal film was deposited to form dual-layer SERS substrate as shown in Figure 4, with the SAM as the dielectric spacer. The procedure can be repeated to form multiple layers of SERSactive surfaces. In first reported results of SAM multilayer SERS enhancement, SAMs were formed on gold-coated SFON substrates. Another layer of gold was then deposited on these SAMs. A solution of Rhodamine 6G was dropcoated and measured on this substrate. For comparison, SERS measurement of the same concentration of the model analyte was done on a SFON SERS substrate with the amount of SERS-active metal film equal to the total amount of metal film deposited on the multilayer substrates. As shown in Figure 5, a 4-layered SERS substrate having 11mercaptoundecanoic acid as the dielectric spacer exhibited SERS EF of $(1.15 \pm 0.06) \times 10^{6}$ which is more than a 15 -fold increase over the SERS EF $\left((7.4 \pm 0.6) \times 10^{4}\right)$ of optimized SFON substrates. Various studies conducted confirmed that SAM multilayer SERS can be used to optimize SERS activities [86]. In a similar study, SAM multilayer SERS enhancement was also applied to SERS nanoprobes, showing that SAM 

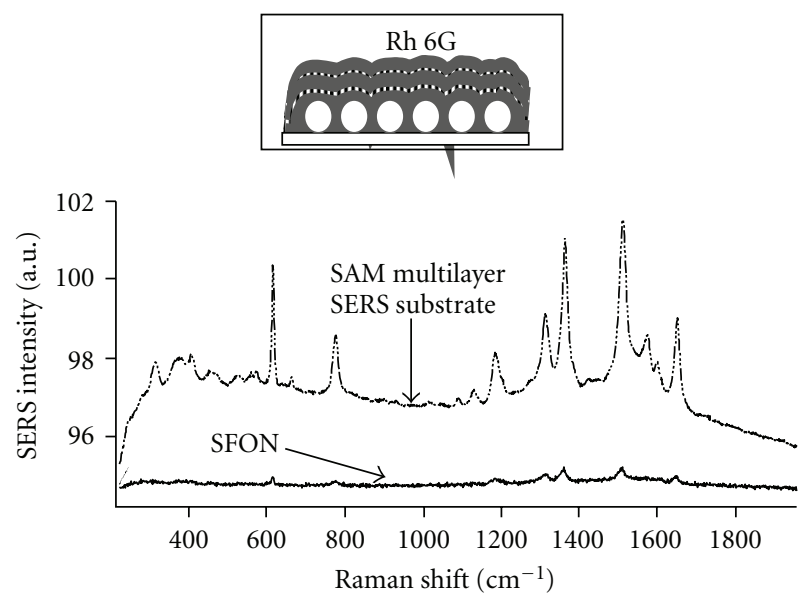

FIGURE 5: SERS spectra of $1 \mu \mathrm{M}$ Rhodamine $6 \mathrm{G}$ measured on SFON and SAM multilayer SERS substrates. Insert is the schematic of SAM multilayer SERS substrates.

multilayer SERS can be applied to different types of SERS substrates $[86,87]$.

Following that observation, a comprehensive investigation on the SAM multilayer SERS substrates enhancement was performed. The results of these studies confirmed current mechanistic understandings of the multilayer SERS enhancement phenomenon. For instance, they clearly revealed the need for the formation of a compact and uniform dielectric spacer for achieving multilayer SERS enhancement. This is because multilayer SERS substrates made from orderly packed SAMs showed larger SERS enhancement relative to those made from SAM with considerable defect sites. That is, when the adjacent SERS-active surfaces are well separated with minimum points of direct contact, it leads to the optimum interaction of the EM fields generated on the SERS-active surfaces and enhances the SERS activity beyond what is expected of conventional SERS substrates. SAM multilayer SERS substrates clearly indicated that SAM can be used to improve the SERS EFs of SERS substrates, thus offering even higher sensitivity for SERSbased analyses. Importantly, the achievable SAM multilayer SERS enhancement can be systematically controlled based on several factors that include the structure of the SAMs and the condition under which the SAMs were formed. Figure 6 shows that the SAM multilayer SERS enhancement can be varied by varying the hydrocarbon chain length of the alkylthiol used to form the SAM dielectric spacer. In this case three types of carboxylic-acid-terminated alkylthiols having 2,11 , or 16 carbon atoms in their hydrocarbon chain lengths were used as dielectric spacers. When benzoic acid was analyzed on these SAM multilayer SERS substrates, there was chain length-dependent SERS enhancement. The chain length-dependent SERS enhancement was attributed to the more uniform SAM formed by the longer hydrocarbon chains $[85,86,88]$.

Apart from the hydrocarbon chain length, it was shown that terminal functional group can be used to vary the dielectric constant of the dielectric spacer and hence control the SERS enhancement. The conditions under which the SAM dielectric spacers are deposited on substrate were also used to control the SERS enhancement. For example, the solvent used for SAM preparation can significantly affect the orderly packing of the SAM. Polar solvents which exhibit less attractive interactions toward the nonpolar hydrocarbon chain can lead to an orderly packed SAM and therefore larger SERS enhancement. The result shown in Figure 7 is spectra of 1, 2-Bis(2-pyridyl)ethylene (BPE) measured on SAM multilayer SERS substrates which were fabricated using the same amount of SERS-active metal film and type of SAM dielectric spacer. However, the $\mathrm{pH}$ under which the monolayers were formed was varied. The variation of the $\mathrm{pH}$ had a tremendous effect on the SAM multilayer SERS enhancement. Apparently, the carboxylic acid-terminated SAM dielectric spacer used in this case formed more carboxylate ions under basic conditions. As result, there were relative repulsive interactions among the carboxylate ions, leading to defects in the dielectric spacer which affected the SERS enhancement. This became obvious when alkylterminated SAM used in similar study showed no preference to the $\mathrm{pH}$ condition in terms of the SERS enhancement $[85,88,89]$.

SAM multilayer SERS offers other unique characteristics which can be exploited to improve analytical capabilities of SERS. As indicated by the arrows in the spectra in Figure 6, the SERS fingerprint of the underlying SAM appears together with the SERS bands of the analyte (benzoic acid). This suggests that the SAM can be used as an internal standard to normalize the SERS intensity of the analytes, thereby improving SERS signal reproducibility. SAM multilayer SERS offers another unique advantage of substrate modification relative to other SAM-modified SERS substrates. That is, SAM multilayer fabrication involves the capping of the SAM with SERS-active metal films. Thus, the entire SERSactive surface is still accessible to the analyte, making SAM multilayer SERS substrate more sensitive relative to other SAM-modified substrates. In effect, it has been shown that multilayer SERS substrate can be greatly optimized in a predictive manner by appropriate choice of the SAM chain length, the tail terminal group, and the solvent used for SAM formation $[85,86]$.

Long-term stability (especially for silver-coated substrates) and reproducibility have been the traditional issues with SERS. The effect of the introduction of the dielectric spacer between adjacent metal films on the substrate stability was therefore considered in the multilayer substrates. Studies conducted with both gold- and silver-coated multilayer SERS substrates showed that the stability and reversibility of the SERS substrates were largely dependent on the metal film overlayer. Both SFON and multilayer substrates lost their SERS activities over the same period of time in a similar manner. It was also possible to reuse the both sets of substrates the same number of times before losing significant level of SERS signals. Thus, the introduction of the SAM dielectric spacer does not significantly affect the long-term stability of the multilayer SERS substrates [82, 86, 90]. It is widely accepted that surface morphology of the substrate plays a significant role in the SERS activity of the SERS substrates as well as the 


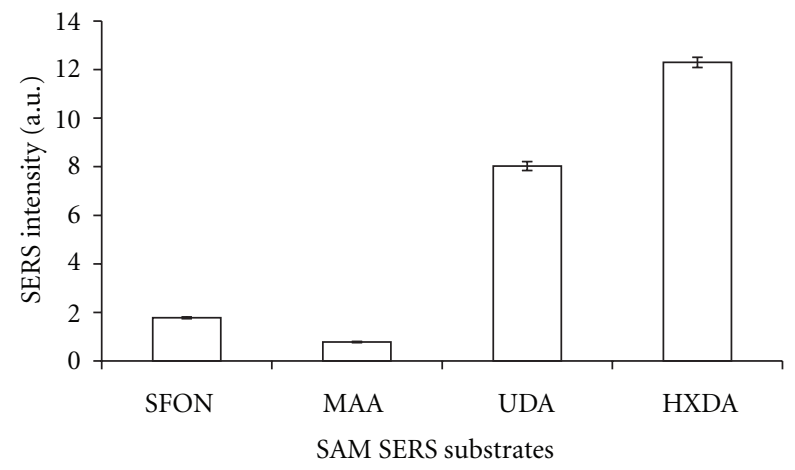

(a)

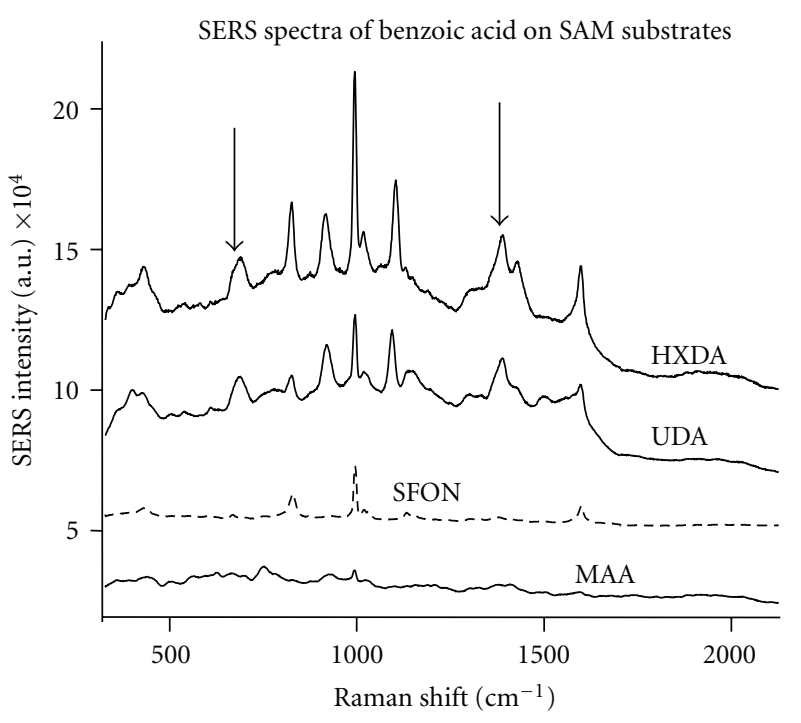

(b)

FIgURE 6: (a) The relative SERS intensities $1 \mathrm{mM}$ benzoic acid obtained on dual-layer coated substrates separated by different types of selfassembled monolayers (MAA, UDA, and HXDA represent 2, 11, and 16 carbon chains, resp.) and optimized SFON substrates. (b) The spectra showing the $1000 \mathrm{~cm}^{-1}$ band of $1 \mathrm{mM}$ benzoic acid used for the measurement.

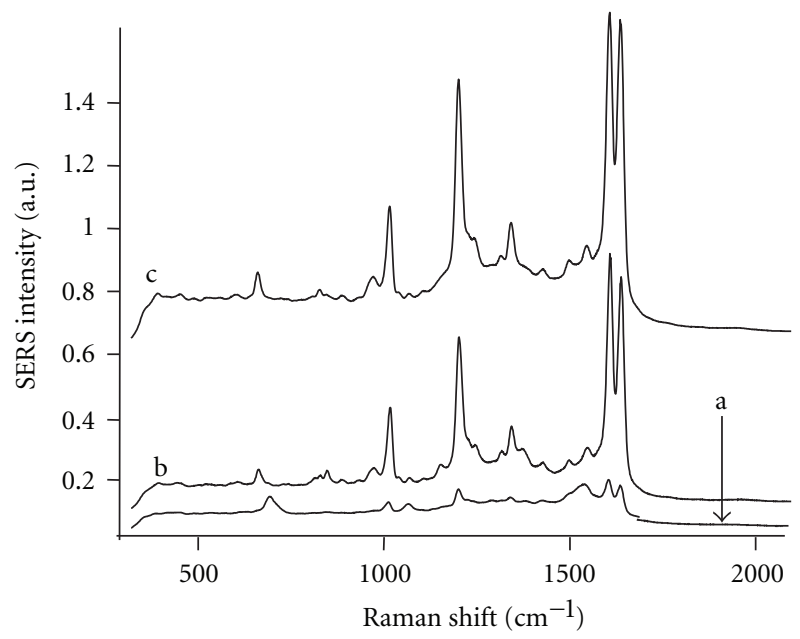

FIGURE 7: A comparison of the SERS intensities of $0.126 \mathrm{mM} \mathrm{BPE}$ on - $\mathrm{COOH}$ - terminated SAM multilayer SERS substrates fabricated in $\mathrm{pH}$ (a) 12, (b) 7.8, and (c) 2 .

reproducibility of their SERS measurements. For the MFON SERS substrates, the surface morphology depends on the nanostructures beneath the metal film and the substructures on these metal-coated nanostructures [91]. Studies have shown that roughness due to the nanostructures was not significantly affected by the introduction of the dielectric spacers $[86,90]$. For this reason, attention was given to the effect of the dielectric spacer on the substructural features on the individual nanostructures. AFM images of typical SFON and SAM multilayer substrates in Figure 8 show the substructures on the individual nanostructures. The roughness values were estimated by drawing several horizontal

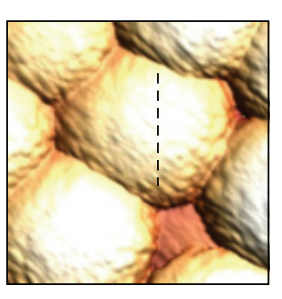

(a)

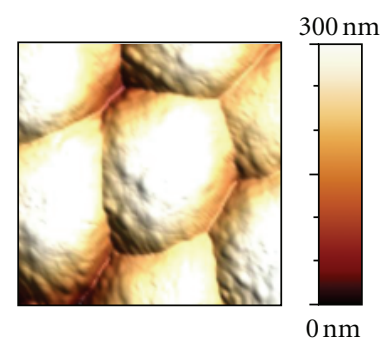

(b)
FIGURE 8: AFM images of (a) SFON and (b) octadecanethiol multilayer SERS substrates showing substructural features. The individual nanostructures were $390 \mathrm{~nm}$ in diameter and the scan size was $800 \mathrm{~nm}$ by $800 \mathrm{~nm}$.

and vertical lines across these individual nanostructures. The average roughness values for the SFON substrates and SAM multilayer SERS substrate were $3.1 \pm 0.6$ and $2.8 \pm 0.7$, respectively, showing that there was no significant effect on the sub-structural features relative to the SFON substrates irrespective of the introduction of the SAM. Remarkably, the relative standard deviation of multilayer SERS measurement was less than half that of the SFON substrates, indicating an improvement in reproducibility. Although the reason for the improved reproducibility is still being investigated, multilayer SERS has clearly illustrated a number of improvements that can be made on any SERS substrates. Thus, SERS activity (i.e., enhancement factors) of SERS substrates can be increased by as much as 20 -fold and their reproducibility improved by using multiple layers of SERS-active metal films interspaced with appropriate dielectric spacers [89]. Using an appropriate SAM, the SERS fingerprints of the dielectric 
spacer can serve as internal standards to minimize spot-tospot variation of SERS signals on a substrate. Importantly, multilayer SERS has demonstrated that SERS substrate can be optimized in various ways without compromising the existing substrate qualities.

\section{Conclusion}

This paper provides an overview of the various ways by which conventional SERS substrates can be optimized in order to achieve enhanced analytical performance. SAMs play an important role in SERS substrates optimization and adaptation for various analytical purposes. Using SAM multilayer SERS substrates, SERS can be optimized to provide SERS enhancement about 20-fold larger than that derived from conventional SERS substrates, thereby improving the sensitivity of the SERS method of analysis. Apart from improving SERS activity, SAMs can also be used to improve long-term stability, reproducibility, and selectivity of the SERS substrates. They provide excellent routes for functionalization of SERS substrates and introduction of internal standard for selective, specific, and reproducible analyte detection in complex samples. The increasing research attention that SERS is attracting is leading to the development of various types of SERS-active platforms for SERS measurement. However, the determining factor in the eventual application of these platforms will be determined on the basis of how their SERS activities can be optimized without losing other desirable SERS substrates properties as well as how the substrates can be tailored for specific applications.

\section{Acknowledgments}

This work was supported by University of Maryland, Baltimore County and US Army Research Office.

\section{References}

[1] M. Moskovits, "Surface-enhanced spectroscopy," Reviews of Modern Physics, vol. 57, no. 3, pp. 783-826, 1985.

[2] K. Kneipp, H. Kneipp, I. Itzkan, R. R. Dasari, and M. S. Feld, "Ultrasensitive chemical analysis by Raman spectroscopy," Chemical Reviews, vol. 99, no. 10, pp. 2957-2975, 1999.

[3] E. C. Le Ru, E. Blackie, M. Meyer, and P. G. Etchegoint, "Surface enhanced raman scattering enhancement factors: a comprehensive study," Journal of Physical Chemistry C, vol. 111, no. 37, pp. 13794-13803, 2007.

[4] K. Kneipp, Y. Wang, H. Kneipp et al., "Single molecule detection using surface-enhanced Raman scattering (SERS)," Physical Review Letters, vol. 78, no. 9, pp. 1667-1670, 1997.

[5] S. M. Nie, "Probing single molecules and single nanoparticles by surface- enhanced Raman scattering," Abstracts of Papers American Chemical Society, vol. 221, p. 110-PHYS, 2001.

[6] S. Nie and S. R. Emory, "Probing single molecules and single nanoparticles by surface-enhanced Raman scattering," Science, vol. 275, no. 5303, pp. 1102-1106, 1997.

[7] M. Fleischmann, P. J. Hendra, and A. J. McQuillan, "Raman spectra of pyridine adsorbed at a silver electrode," Chemical Physics Letters, vol. 26, no. 2, pp. 163-166, 1974.
[8] A. J. McQuillan, "The discovery of surface-enhanced Raman scattering," Notes and Records of the Royal Society, vol. 63, no. 1, pp. 105-109, 2009.

[9] D. L. Jeanmaire and R. P. Van Duyne, "Surface Raman spectroelectrochemistry Part I. Heterocyclic, aromatic, and aliphatic amines adsorbed on the anodized silver electrode," Journal of Electroanalytical Chemistry, vol. 84, no. 1, pp. 1-20, 1977.

[10] M. G. Albrecht and J. A. Creighton, "Anomalously intense Raman spectra of pyridine at a silver electrode," Journal of the American Chemical Society, vol. 99, no. 15, pp. 5215-5217, 1977.

[11] R. L. Garrell, "Surface-enhanced Raman spectroscopy," Analytical Chemistry, vol. 61, no. 6, pp. 401A-411A, 1989.

[12] E. J. Zeman and G. C. Schatz, "An accurate electromagnetic theory study of surface enhancement factors for silver, gold, copper, lithium, sodium, aluminum, gallium, indium, zinc, and cadmium," The Journal of Physical Chemistry, vol. 91, no. 3, pp. 634-643, 1987.

[13] A. M. Alak and T. Vo-Dinh, "Surface-enhanced Raman spectrometry of organophosphorus chemical agents," Analytical Chemistry, vol. 59, no. 17, pp. 2149-2153, 1987.

[14] P. D. Enlow, M. Buncick, R. J. Warmack, and T. Vo-Dinh, "Detection of nitro polynuclear aromatic compounds by surface-enhanced Raman spectrometry," Analytical Chemistry, vol. 58, no. 6, pp. 1119-1123, 1986.

[15] V. A. Narayanan, J. M. Bello, D. L. Stokes, and T. Vo-Dinh, "Surface-enhanced raman analysis of vitamin B complex: quantitative detection of p-aminobenzoic acid," Journal of Raman Spectroscopy, vol. 22, pp. 327-331, 1991.

[16] N. Taranenko, J. P. Alarie, D. L. Stokes, and T. Vo-Dinh, "Surface-enhanced Raman detection of nerve agent simulant (DMMP and DIMP) vapor on electrochemically prepared silver oxide substrates," Journal of Raman Spectroscopy, vol. 27, no. 5, pp. 379-384, 1996.

[17] T. Vo-Dinh, L. R. Allain, and D. L. Stokes, "Cancer gene detection using surface-enhanced Raman scattering (SERS)," Journal of Raman Spectroscopy, vol. 33, no. 7, pp. 511-516, 2002.

[18] N. R. Isola, D. L. Stokes, and T. Vo-Dinh, "Surface-rnhanced Raman gene probe for HIV detection," Analytical Chemistry, vol. 70, no. 7, pp. 1352-1356, 1998.

[19] D. A. Stuart, J. M. Yuen, N. Shah et al., "In vivo glucose measurement by surface-enhanced Raman spectroscopy," Analytical Chemistry, vol. 78, no. 20, pp. 7211-7215, 2006.

[20] X. Zhang, M. A. Young, O. Lyandres, and R. P. Van Duyne, "Rapid detection of an anthrax biomarker by surfaceenhanced Raman spectroscopy," Journal of the American Chemical Society, vol. 127, no. 12, pp. 4484-4489, 2005.

[21] D. A. Stuart, C. R. Yonzon, X. Zhang et al., "Glucose sensing using near-infrared surface-enhanced raman spectroscopy: gold surfaces, 10-day stability, and improved accuracy," Analytical Chemistry, vol. 77, no. 13, pp. 4013-4019, 2005.

[22] D. A. Stuart, K. B. Biggs, and R. P. Van Duyne, "Surfaceenhanced Raman spectroscopy of half-mustard agent," Analyst, vol. 131, no. 4, pp. 568-572, 2006.

[23] J. Kneipp, H. Kneipp, M. McLaughlin, D. Brown, and K. Kneipp, "In vivo molecular probing of cellular compartments with gold nanoparticles and nanoaggregates," Nano Letters, vol. 6, no. 10, pp. 2225-2231, 2006.

[24] H. Li, J. Sun, T. Alexander, and B. M. Cullum, "Implantable SERS nanosensors for pre-symptomatic detection of BW 
agents," in Chemical and Biological Sensing VI, vol. 5795 of Proceedings of the SPIE, pp. 8-18, March 2005.

[25] H. Li, C. E. Baum, and B. M. Cullum, "Label-free detection of antigens using implantable sers nanosensors," in Smart Medical and Biomedical Sensor Technology III, vol. 6007 of Proceedings of the SPIE, pp. 58-70, October 2005.

[26] C. E. Talley, L. Jusinski, C. W. Hollars, S. M. Lane, and T. Huser, "Intracellular $\mathrm{pH}$ sensors based on surface-enhanced raman scattering," Analytical Chemistry, vol. 76, no. 23, pp. 70647068, 2005.

[27] M. Fan, G. F. S. Andrade, and A. G. Brolo, "A review on the fabrication of substrates for surface enhanced Raman spectroscopy and their applications in analytical chemistry," Analytica Chimica Acta, vol. 693, no. 1-2, pp. 7-25, 2011.

[28] X. M. Lin, Y. Cui, Y. H. Xu, B. Ren, and Z. Q. Tian, "Surfaceenhanced raman spectroscopy: substrate-related issues," Analytical and Bioanalytical Chemistry, vol. 394, no. 7, pp. 1729$1745,2009$.

[29] K. Hering, D. Cialla, K. Ackermann et al., "SERS: a versatile tool in chemical and biochemical diagnostics," Analytical and Bioanalytical Chemistry, vol. 390, no. 1, pp. 113-124, 2008.

[30] T. Vo-Dinh, "Surface-enhanced Raman spectroscopy using metallic nanostructures," Trends in Analytical Chemistry, vol. 17, no. 8-9, pp. 557-582, 1998.

[31] G. A. Baker and D. S. Moore, "Progress in plasmonic engineering of surface-enhanced Raman-scattering substrates toward ultra-trace analysis," Analytical and Bioanalytical Chemistry, vol. 382, no. 8, pp. 1751-1770, 2005.

[32] K. L. Kelly, E. Coronado, L. L. Zhao, and G. C. Schatz, "The optical properties of metal nanoparticles: the influence of size, shape, and dielectric environment," Journal of Physical Chemistry B, vol. 107, no. 3, pp. 668-677, 2003.

[33] S. R. Emory and S. Nie, "Near-field surface-enhanced Raman spectroscopy on single silver nanoparticles," Analytical Chemistry, vol. 69, no. 14, pp. 2631-2635, 1997.

[34] A. R. Bizzarri and S. Cannistraro, "SERS detection of thrombin by protein recognition using functionalized gold nanoparticles," Nanomedicine, vol. 3, no. 4, pp. 306-310, 2007.

[35] W. E. Doering and S. Nie, "Spectroscopic tags using dyeembedded nanoparticles and surface-enhanced Raman scattering," Analytical Chemistry, vol. 75, no. 22, pp. 6171-6176, 2003.

[36] M. Baia, F. Toderas, L. Baia, D. Maniu, and S. Astilean, "Multilayer structures of self-assembled gold nanoparticles as a unique SERS and SEIRA substrate," ChemPhysChem, vol. 10, no. 7, pp. 1106-1111, 2009.

[37] J. L. Seung, M. B. Jeong, and M. Moskovits, "Polarizationdependent surface-enhanced raman scattering from a silvernanoparticle-decorated single silver nanowire," Nano Letters, vol. 8, no. 10, pp. 3244-3247, 2008.

[38] H. Wang, J. Kundu, and N. J. Halas, "Plasmonic nanoshell arrays combine surface-enhanced vibrational spectroscopies on a single substrate," Angewandte Chemie, vol. 46, no. 47, pp. 9040-9044, 2007.

[39] M. Kahraman, M. M. Yazici, F. Şahin, and M. Çulha, "Convective assembly of bacteria for surface-enhanced Raman scattering," Langmuir, vol. 24, no. 3, pp. 894-901, 2008.

[40] M. A. De Jesús, K. S. Giesfeldt, J. M. Oran, N. A. AbuHatab, N. V. Lavrik, and M. J. Sepaniak, "Nanofabrication of densely packed metal-polymer arrays for surface-enhanced raman spectrometry," Applied Spectroscopy, vol. 59, no. 12, pp. 1501-1508, 2005.

[41] N. A. Abu Hatab, J. M. Oran, and M. J. Sepaniak, "Surfaceenhanced Raman spectroscopy substrates created via electron beam lithography and nanotransfer printing," ACS Nano, vol. 2, no. 2, pp. 377-385, 2008.

[42] H. Im, K. C. Bantz, N. C. Lindquist, C. L. Haynes, and S. H. Oh, "Vertically oriented sub-10-nm plasmonic nanogap arrays," Nano Letters, vol. 10, no. 6, pp. 2231-2236, 2010.

[43] C. L. Haynes and R. P. Van Duyne, "Nanosphere lithography: a versatile nanofabrication tool for studies of size-dependent nanoparticle optics," Journal of Physical Chemistry B, vol. 105, no. 24, pp. 5599-5611, 2001.

[44] M. D. Malinsky, K. Lance Kelly, G. C. Schatz, and R. P. van Duyne, "Nanosphere lithography: effect of substrate on the localized surface plasmon resonance spectrum of silver nanoparticles," Journal of Physical Chemistry B, vol. 105, no. 12, pp. 2343-2350, 2001.

[45] J. C. Hulteen, D. A. Treichel, M. T. Smith, M. L. Duval, T. R. Jensen, and R. P. Van Duyne, "Nanosphere lithography: sizetunable silver nanoparticle and surface cluster arrays," Journal of Physical Chemistry B, vol. 103, no. 19, pp. 3854-3863, 1999.

[46] J. M. Bello, D. L. Stokes, and T. Vo-Dinh, "Silver-coated alumina as a new medium for surfaced-enhanced Raman scattering analysis," Applied Spectroscopy, vol. 43, no. 8, pp. 1325-1330, 1989.

[47] M. Litorja, C. L. Haynes, A. J. Haes, T. R. Jensen, and R. P. Van Duyne, "Surface-enhanced Raman scattering detected temperature programmed desorption: optical properties, nanostructure, and stability of silver film over $\mathrm{SiO}_{2}$ nanosphere surfaces," Journal of Physical Chemistry B, vol. 105, no. 29, pp. 69076915, 2001.

[48] J. C. Love, L. A. Estroff, J. K. Kriebel, R. G. Nuzzo, and G. M. Whitesides, "Self-assembled monolayers of thiolates on metals as a form of nanotechnology," Chemical Reviews, vol. 105, no. 4, pp. 1103-1169, 2005.

[49] N. Muskal, I. Turyan, and D. Mandler, "Self-assembled monolayers on mercury surfaces," Journal of Electroanalytical Chemistry, vol. 409, no. 1-2, pp. 131-136, 1996.

[50] M. D. Malinsky, K. L. Kelly, G. C. Schatz, and R. P. Van Duyne, "Chain length dependence and sensing capabilities of the localized surface plasmon resonance of silver nanoparticles chemically modified with alkanethiol self-assembled monolayers," Journal of the American Chemical Society, vol. 123, no. 7, pp. 1471-1482, 2001.

[51] C. E. D. Chidsey and D. N. Loiacono, "Chemical functionality in self-assembled monolayers: structural and electrochemical properties," Langmuir, vol. 6, no. 3, pp. 682-691, 1990.

[52] B. J. Kennedy, S. Spaeth, M. Dickey, and K. T. Carron, "Determination of the distance dependence and experimental effects for modified sers substrates based on self-assembled monolayers formed using alkanethiols," Journal of Physical Chemistry B, vol. 103, no. 18, pp. 3640-3646, 1999.

[53] S. Lal, N. K. Grady, G. P. Goodrich, and N. J. Halas, "Profiling the near field of a plasmonic nanoparticle with Raman-based molecular rulers," Nano Letters, vol. 6, no. 10, pp. 2338-2343, 2006.

[54] C. S. Levin, B. G. Janesko, R. Bardhan, G. E. Scuseria, J. D. Hartgerink, and N. J. Halas, "Chain-length-dependent vibrational resonances in alkanethiol self-assembled monolayers observed on plasmonic nanoparticle substrates," Nano Letters, vol. 6, no. 11, pp. 2617-2621, 2006.

[55] M. Tsen and L. Sun, "Surface-enhanced Raman scattering from functionalized self-assembled monolayers. Part 1. Distance dependence of enhanced Raman scattering from a terminal phenyl group," Analytica Chimica Acta, vol. 307, no. 2-3, pp. 333-340, 1995. 
[56] M. Rycenga, Z. Wang, E. Gordon et al., "Probing the photothermal effect of gold-based nanocages with surfaceenhanced Raman scattering (SERS)," Angewandte Chemie, vol. 48, no. 52, pp. 9769-9927, 2009.

[57] M. Moskovits and J. S. Suh, "Conformation of mono- and dicarboxylic acids adsorbed on silver surfaces," Journal of the American Chemical Society, vol. 107, no. 24, pp. 6826-6829, 1985.

[58] K. E. Brown and D. D. Dlott, "High-pressure raman spectroscopy of molecular monolayers adsorbed on a metal surface," Journal of Physical Chemistry C, vol. 113, no. 14, pp. 5751-5757, 2009.

[59] C. W. Meuse, G. Niaura, M. L. Lewis, and A. L. Plant, "Assessing the molecular structure of alkanethiol monolayers in hybrid bilayer membranes with vibrational spectroscopies," Langmuir, vol. 14, no. 7, pp. 1604-1611, 1998.

[60] M. A. Bryant and J. E. Pemberton, "Surface Raman scattering of self-assembled monolayers formed from 1-alkanethiols: behavior of films at Au and comparison to films at Ag," Journal of the American Chemical Society, vol. 113, no. 22, pp. 82848293, 1991.

[61] L. Guerrini, I. Izquierdo-Lorenzo, R. Rodriguez-Oliveros et al., " $\alpha, \omega$-aliphatic diamines as molecular linkers for engineering Ag nanoparticle clusters: tuning of the interparticle distance and sensing application," Plasmonics, vol. 5, no. 3, pp. 273286, 2010.

[62] M. Culha, D. Stokes, L. R. Allain, and T. Vo-Dinh, "Surfaceenhanced Raman scattering substrate based on a selfassembled monolayer for use in gene diagnostics," Analytical Chemistry, vol. 75, no. 22, pp. 6196-6201, 2003.

[63] M. D. Porter, R. J. Lipert, L. M. Siperko, G. Wang, and R. Narayanan, "SERS as a bioassay platform: fundamentals, design, and applications," Chemical Society Reviews, vol. 37, no. 5, pp. 1001-1011, 2008.

[64] K. J. Yoon, H. K. Seo, H. Hwang et al., "Bioanalytical application of SERS immunoassay for detection of prostate-specific antigen," Bulletin of the Korean Chemical Society, vol. 31, no. 5, pp. 1215-1218, 2010.

[65] J. D. Driskell, R. J. Lipert, and M. D. Porter, "Labeled gold nanoparticles immobilized at smooth metallic substrates: systematic investigation of surface plasmon resonance and surface-enhanced raman scattering," Journal of Physical Chemistry B, vol. 110, no. 35, pp. 17444-17451, 2006.

[66] D. S. Grubisha, R. J. Lipert, H. Y. Park, J. Driskell, and M. D. Porter, "Femtomolar detection of prostate-specific antigen: an immunoassay based on surface-enhanced Raman scattering and immunogold labels," Analytical Chemistry, vol. 75, no. 21, pp. 5936-5943, 2003.

[67] H. Li, J. Sun, and B. M. Cullum, "Label-free detection of proteins using SERS-based immuno-nanosensors," Nanobiotechnology, vol. 2, no. 1-2, pp. 17-28, 2006.

[68] H. Li, J. Sun, and B. M. Cullum, "Nanosphere-based SERS immuno-sensors for protein analysis," in Smart Medical and Biomedical Sensor Technology II, vol. 5588 of Proceedings of the SPIE, pp. 19-30, October 2004.

[69] C. R. Yonzon, C. L. Haynes, X. Zhang, J. T. Walsh, and R. P. Van Duyne, "A glucose biosensor based on surface-enhanced raman scattering: improved partition layer, temporal stability, reversibility, and resistance to serum protein interference," Analytical Chemistry, vol. 76, no. 1, pp. 78-85, 2004.

[70] G. L. De Vault and M. J. Sepaniak, "Spatially focused deposition of capillary electrophoresis effluent onto surfaceenhanced Raman-active substrates for off-column spectroscopy," Electrophoresis, vol. 22, no. 11, pp. 2303-2311, 2001.
[71] O. Neumann, D. Zhang, F. Tam, S. Lal, P. Wittung-Stafshede, and N. J. Halas, "Direct optical detection of aptamer conformational changes induced by target molecules," Analytical Chemistry, vol. 81, no. 24, pp. 10002-10006, 2009.

[72] J. C. S. Costa, A. C. Sant'Ana, P. Corio, and M. L. A. Temperini, "Chemical analysis of polycyclic aromatic hydrocarbons by surface-enhanced Raman spectroscopy," Talanta, vol. 70, no. 5, pp. 1011-1016, 2006.

[73] T. O. Deschaines and K. T. Carron, "Stability and surface uniformity of selected thiol-coated SERS surfaces," Applied Spectroscopy, vol. 51, no. 9, pp. 1355-1359, 1997.

[74] M. Culha, D. Stokes, L. R. Allain, and T. Vo-Dinh, "Surfaceenhanced Raman scattering substrate based on a selfassembled monolayer for use in gene diagnostics," Analytical Chemistry, vol. 75, no. 22, pp. 6196-6201, 2003.

[75] M. Culha, D. Stokes, and T. Vo-Dinh, "Surface-enhanced Raman scattering for cancer diagnostics: detection of the BCL2 gene," Expert Review of Molecular Diagnostics, vol. 3, no. 5, pp. 669-675, 2003.

[76] T. O. Deschaines and K. T. Carron, "Stability and surface uniformity of selected thiol-coated SERS surfaces," Applied Spectroscopy, vol. 51, no. 9, pp. 1355-1359, 1997.

[77] A. Lorén, J. Engelbrektsson, C. Eliasson et al., "Internal standard in surface-enhanced Raman spectroscopy," Analytical Chemistry, vol. 76, no. 24, pp. 7391-7395, 2004.

[78] J. C. S. Costa, A. C. Sant'Ana, P. Corio, and M. L. A. Temperini, "Chemical analysis of polycyclic aromatic hydrocarbons by surface-enhanced Raman spectroscopy," Talanta, vol. 70, no. 5, pp. 1011-1016, 2006.

[79] D. Zhang, Y. Xie, S. K. Deb, V. J. Davison, and D. Ben-Amotz, "Isotope edited internal standard method for quantitative surface-enhanced raman spectroscopy," Analytical Chemistry, vol. 77, no. 11, pp. 3563-3569, 2005.

[80] S. E. J. Bell, J. N. Mackle, and N. M. S. Sirimuthu, "Quantitative surface-enhanced Raman spectroscopy of dipicolinic acid-towards rapid anthrax endospore detection," Analyst, vol. 130, no. 4, pp. 545-549, 2005.

[81] P. Kao, N. A. Malvadkar, M. Cetinkaya, H. Wang, D. L. Allara, and M. C. Demirel, "Surface-enhanced raman detection on metalized nanostructured poly(p-xylylene) films," Advanced Materials, vol. 20, no. 18, pp. 3562-3565, 2008.

[82] H. Li and B. M. Cullum, "Dual layer and multilayer enhancements from silver film over nanostructured surface-enhanced Raman substratess," Applied Spectroscopy, vol. 59, no. 4, pp. 410-417, 2005.

[83] H. Li, C. E. Baum, J. Sun, and B. M. Cullum, "Multilayer enhanced SERS active materials: fabrication, characterization, and application to trace chemical detection," in Chemical and Biological Sensing VII, vol. 6218 of Proceedings of the SPIE, April 2006.

[84] H. Li, P. H. Patel, and B. M. Cullum, "Novel multilayered SERS substrates for trace chemical and biochemical analysis," in Smart Medical and Biomedical Sensor Technology II, vol. 5588 of Proceedings of the SPIE, pp. 87-97, October 2004.

[85] C. K. Klutse, H. Li, and B. M. Cullum, "Optimization of multilayer Surface Enhanced Raman Scattering (SERS):Immunonanosensors via self-assembled monolayer spacers," in Smart Biomedical and Physiological Sensor Technology VI, vol. 7313 of Proceedings of the SPIE, April 2009.

[86] B. M. Cullum, H. Li, M. V. Schiza, and M. E. Hankus, "Characterization of multilayer-enhanced surface-enhanced Raman scattering (SERS) substrates and their potential for SERS nanoimaging," Nanobiotechnology, vol. 3, no. 1, pp. 111, 2007. 
[87] M. E. Hankus, H. Li, G. J. Gibson, and B. M. Cullum, "Surface-enhanced Raman scattering-based nanoprobe for high-resolution, non-scanning chemical imaging," Analytical Chemistry, vol. 78, no. 21, pp. 7535-7546, 2006.

[88] C. K. Klutse and B. M. Cullum, "Development of SAMbased multi-layer SERS substrates for intracellular analyses," in Smart Biomedical and Physiological Sensor Technologies VII, vol. 7674 of Proceedings of the SPIE, April 2010.

[89] C. K. Klutse and B. M. Cullum, "Optimization of SAM-based multilayer SERS substrates for intracellular analyses: the effect of terminating functional groups," in Micro-sensors for Cellular Analyses, vol. 8025 of Proceedings of the SPIE, 2011.

[90] H. Li, C. E. Baum, J. Sun, and B. M. Cullum, "Multilayer enhanced gold film over nanostructure surface-enhanced Raman," Applied Spectroscopy, vol. 60, no. 12, pp. 1377-1385, 2006.

[91] L. A. Dick, A. D. McFarland, C. L. Haynes, and R. P. Van Duyne, "Metal film over nanosphere (MFON) electrodes for surface-enhanced Raman spectroscopy (SERS): improvements in surface nanostructure stability and suppression of irreversible loss," Journal of Physical Chemistry B, vol. 106, no. 4, pp. 853-860, 2002. 

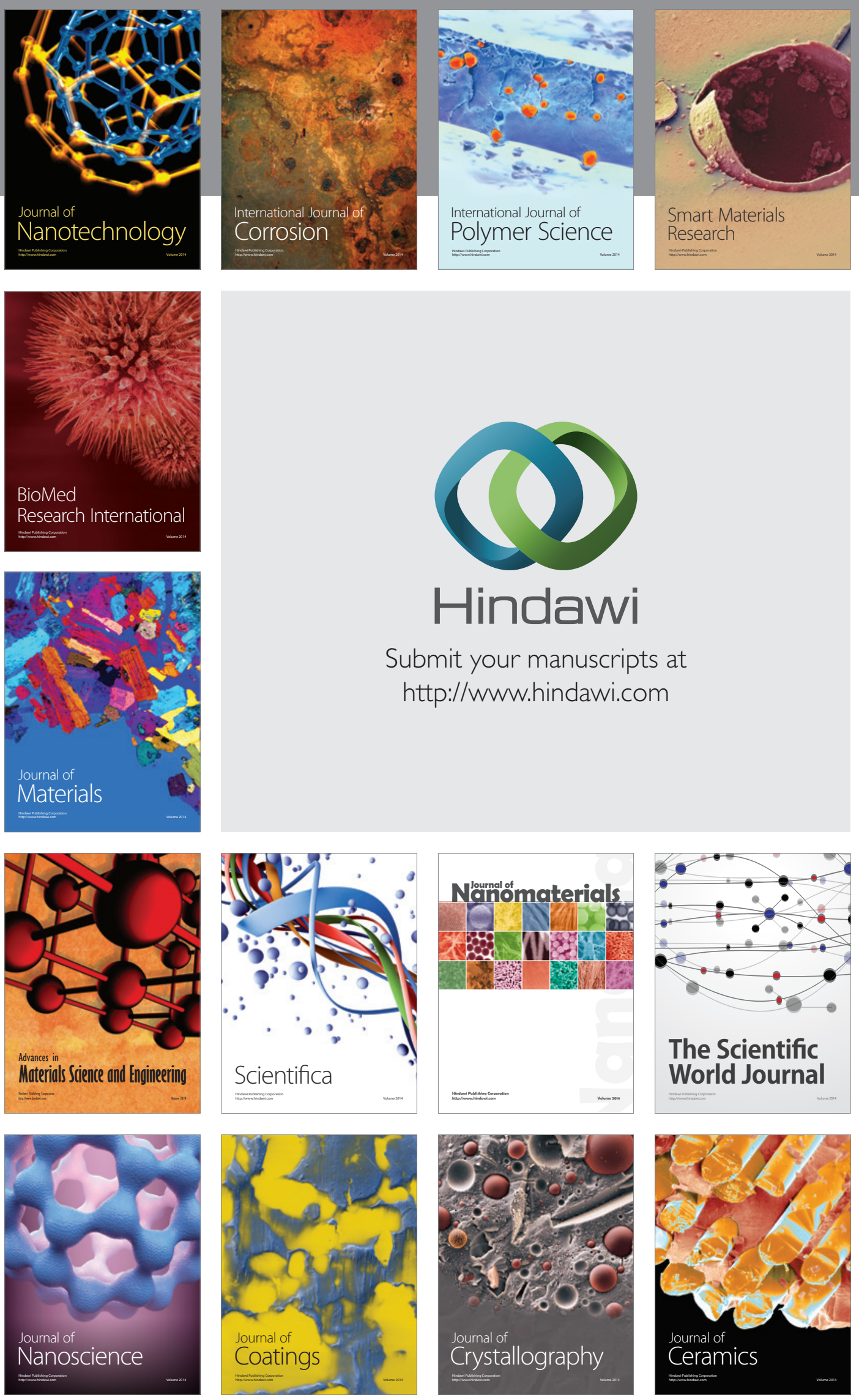

The Scientific World Journal

Submit your manuscripts at

http://www.hindawi.com

\section{World Journal}

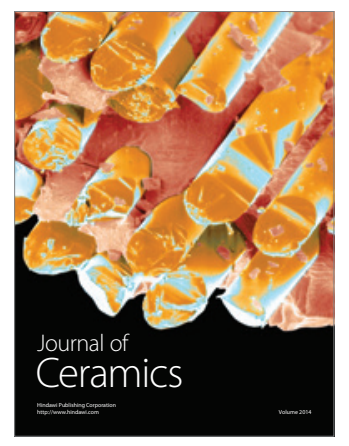

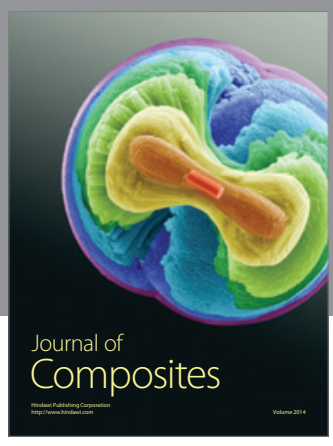
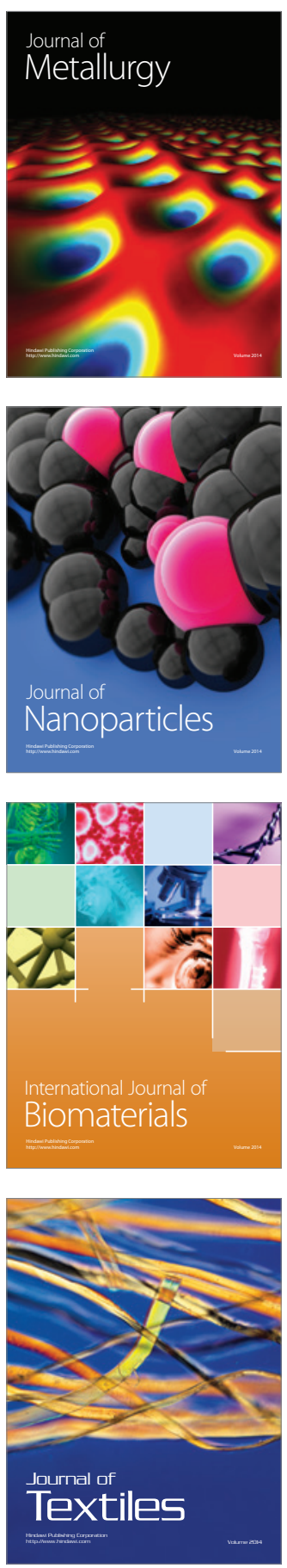